\title{
First interferometric observations in Irbene - Torun baseline conducted by VIRAC
}

\author{
V. Bezrukovs ${ }^{a}$, J. Shteinbergs ${ }^{a}$, I. Shmeld ${ }^{a}$, A. Aberfelds ${ }^{a}$, M. Bleiders ${ }^{a}$, \\ A. Orbidans ${ }^{a}$, K. Skirmante ${ }^{a}$, M. Gawronski ${ }^{b}$, R. Feiler ${ }^{b}$ \\ a) Engineering Research Institute "Ventspils International Radio Astronomy Centre" of Ventspils \\ University of Applied Sciences \\ Inzenieru Street 101, Ventspils, LV-3601, Latvia \\ b) Torun Centre for Astronomy, Nicolaus Copernicus University \\ Gagarina street 11 87-100, Torun, Poland \\ E-mail:vladislavsb@venta.lv
}

\begin{abstract}
Ventspils International Radio Astronomy Centre (VIRAC, Latvia) operates two radio telescopes, RT-16 and RT-32 accordingly, with fully steerable 16- and 32- $m$ Cassegrain type antennas. The main receiving systems of the both telescopes are cryogenic receivers with 4.5-8.8 GHz frequency range. Additionally, in 2019 radio telescope RT-32 will be equipped with a new $\mathrm{L}$ band receiver. Data registration units of the both antennas are suitable for interferometric observations. The Nicolaus Copernicus University Department of Radio Astronomy in Torun, Poland, operates a 32$m$ radio telescope RT-4, which also works in similar bands - L, C and M - and regularly participates in the VLBI observations. Moreover, VIRAC possesses a high-performance computer cluster with SFXC software correlator developed at JIVE. In the spring 2018, VIRAC team with support from Torun group conducted several VLBI experiments in the baseline Irbene-Torun using the Irbene RT-16 radio telescope and Torun RT-4 radio telescope. Successful fringes and FITS files with obtained $u v$ data implies that data correlation and post processing facilities established in VIRAC allow to run small-scale interferometric tasks.
\end{abstract}

Keywords: VIRAC, radio astronomy, galactic masers, VLBI observations, SFXC, correlation 


\section{Introduction}

The radio telescopes RT-32 and RT-16 in Irbene, operated by VIRAC, are equipped with broad band (frequency range 4.5-8.8 GHz) receiving systems and a modern VLBI data acquisition system [1]. The first fringes in the European VLBI Network (EVN) Network Monitoring Experiments were detected in April of 2012. This demonstrated ability of Irbene radio observatory to participate in the EVN as a VLBI station [2]. Since then, VIRAC staff have been gradually improving the observation process, including investments in the infrastructure, software development and refining VLBI data processing tools. Recent software solutions for VLBI and accessibility to High Performance Computers (HPC) allow to process large amount of radio astronomical data, typically 1 to 4 Gbps per station, in several data flows utilizing relatively small data processing centres, e.g. data processing centre established in VIRAC.

\section{VLBI experiment scheduling and data processing pipeline}

Typical organization of a VLBI observation session includes the following steps: experiment planning and telescope time scheduling; performing radio astronomical observations under automatic control of the Field System (FS) [3]; data acquisition and storage; data correlation and post-correlation data processing. Figure 1 shows the VLBI data processing pipeline implemented in VIRAC.

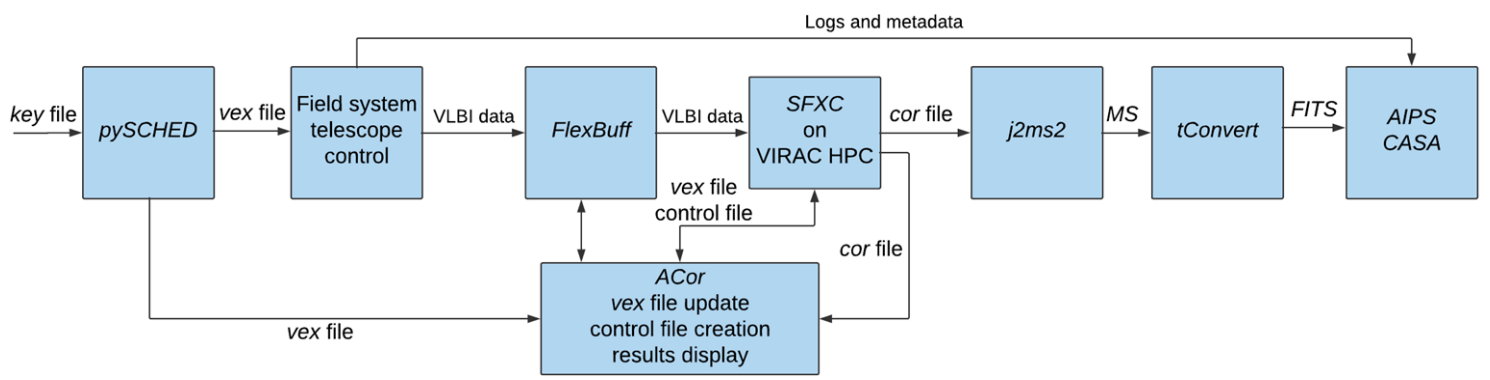

Figure 1: VLBI data processing pipeline established in VIRAC.

The process begins by creating the key file containing information about the participating stations, sources being observed and timing of planned scans. For observation scheduling VIRAC uses the JIVE (the Joint Institute for VLBI ERIC) pySCHED software. It uses the key file as input and creates a vex file ${ }^{1}$. Thereafter it is loaded into FS for controlling the antenna movement and data acquisition. Observational VLBI data from stations in $V D I F^{2}$ or Mark5a/b formats are delivered to the data storage on VIRAC FlexBuff-1 or FlexBuff-2 servers with 320 TB disk space by help of the Jive $5 a b$ utilities for sending to correlator implemented on VIRAC HPC. Currently VIRAC uses two correlators, self-developed KANA for near field [4] and SFXC (Software FX Correlator), developed by JIVE [5], for far-field sources. VIRAC HPC consists of 30 nodes with two Intel Xeon E5-2630 v3 processors and 128 GB RAM in each node and a 10 Gbps connection to GÉANT ${ }^{3}$.

VIRAC also owns a self-developed Automated Correlation System or ACor, providing following functionality: vex file update, control file creation for $S F X C$ correlator, correlation processing and display of correlation results. ACor system was created using AngularJS framework and the Twitter Bootstrap responsive web interface [6]. To correlate with SFXC, a

\footnotetext{
${ }^{1}$ vex-VLBI Experiment file format.

${ }^{2} V D I F$ - VLBI Data Interchange Format.

${ }^{3}$ GÉANT pan-European network. https://www.geant.org.
} 
correlation control file is prepared and the vex file sections ${ }^{4}$ - TAPELOG_OBS, CLOCK, EOP, BITSTREAMS and THREADS - are updated. Control file contains correlation parameters, i.e. integration time, number of channels, location of data files. The correlation follows by creation of the *.cor file. The jplotter tool, developed by JIVE, is used to inspect the correlation results and to create the inspection plots, for example, station weights as function of time, autocorrelation and cross-correlation amplitudes and phases as functions of channels, and others. In order to use the jplotter, it is necessary to convert the *.cor file into the so-called Measurement Sets $(M S)$. The j2ms 2 tool, developed by JIVE, is used for this purpose. When $M S$ is created, it can be converted to FITS ${ }^{5}$ file using the JIVE $t$ Convert tool. Afterwards, the FITS file can be loaded into the AIPS (Astronomical Image Processing System) or CASA (Common Astronomy Software Applications package) for post-correlation data processing. VIRAC currently uses AIPS package for data editing, calibration, fringe fitting and imaging tasks of the post-correlation data process.

\section{Irbene - Torun interferometric experiment}

During the NEXPReS ${ }^{6}$ project an important collaboration between Irbene and Torun was established to improve VLBI and e-VLBI (real time VLBI) data processing techniques at both observatories. The Torun observatory operates a 32- $m$ radio telescope working in similar bands L, C, M and - and regularly participates in VLBI observations. Between 2012 and 2013, several e-VLBI sessions were organized involving Irbene and Torun antennas and using the DiFX (deployed in $\mathrm{PSNC}^{7}$ ) and SFXC (deployed in VIRAC) correlators. The management of the data processing was mutually taken on by both operators. The data transfer in real-time was tested at multiple configurations of the antennas and the correlators, as well as at a variable number of channels. The results of these experiments demonstrated technical possibility to correlate at least two data flows in real time. Unfortunately, the fringes have not been obtained [2]. Attempts to create a working VLBI data processing pipeline in VIRAC were boosted in years 2017 and 2018 within two scientific projects - ASTRA $^{8}$ and TorIn ${ }^{9}$, related to galactic maser observations in single dish and VLBI mode. In April 2018, short VLBI observations of methanol maser W3(OH) were performed with two radio telescopes: Irbene RT-16 and Torun RT-4. The purpose of the experiment was to practice planning observations and data processing steps. During this experiment, VIRAC team created a schedule for each telescope, collected recorded data on FlexBuff server, carried out data correlation with SFXC correlator and converted measurement sets to the FITS files suitable for subsequent interpretation in the AIPS or CASA. April 26, 2018, saw the first successful VLBI experiment at $6.7 \mathrm{GHz}$ - data correlation returned the first fringes on baseline Irbene-Torun $(530 \mathrm{~km})$. The quasars 3C123 and 3C84 were chosen as calibrators and the target source was the methanol maser $\mathrm{W} 3(\mathrm{OH})$. Figure 2 clearly shows the cross-correlation functions with fringe pattern for this baseline. Figure 3 reveals cross-correlation results of amplitude and phase as function of channels, in the left frame - source $3 \mathrm{C} 84$, in the right frame -

\footnotetext{
${ }^{4}$ vex file sections definition: https://safe.nrao.edu/wiki/bin/view/VLBA/Vex2doc

${ }^{5}$ FITS - Flexible Image Transport System file format https://fits.gsfc.nasa.gov/fits_standard.html.

${ }^{6}$ NEXPReS - Novel EXplorations Pushing Robust e-VLBI Services (FP7 RI 261525), http://www.nexpres.eu.

${ }^{7}$ PSNC - The Poznan Supercomputing and Networking Centre.

${ }^{8}$ European Regional Development Fund Project No. 1.1.1.1/16/A/213 "Physical and chemical processes in the interstellar medium" - ASTRA.

${ }^{9}$ Ventspils University of Applied Sciences project "Research of the possibility of creating a small VLBI array and radio astronomical data processing centre in VIRAC" - TorIn.
} 
source $\mathrm{W} 3(\mathrm{OH})$. The result for source $3 \mathrm{C} 84$ shows coherent phases and amplitude, indicating that a strong quasar is clearly visible. Both antennas were pointed at the source and the signal from the source is coherent. The result for the source $\mathrm{W} 3(\mathrm{OH})$ shows strong maser signal, as expected. The final step of the post-correlation task, usually, is to create an image (FFT of $u v$-plane) of the target source. In this case the session lasted only 30 minutes with one baseline so the total number of visibilities was very small. Therefore, this step was not performed.
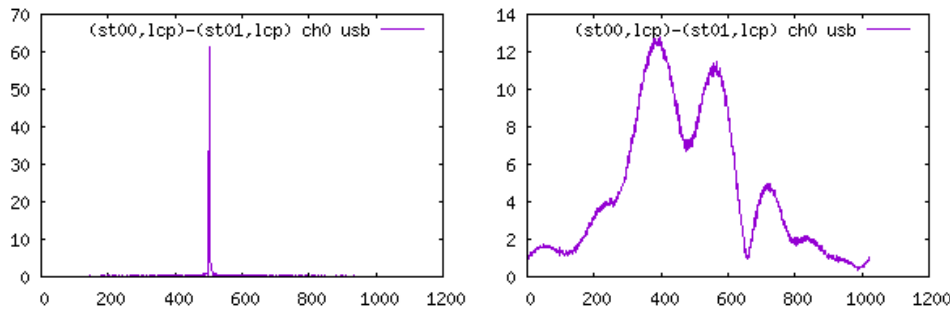

Figure 2: Cross-correlation functions at the baseline Irbene - Torun. Date: 26 Apr 2018, frequency: $6662.49 \mathrm{MHz}$, USB, LCP - LCP: calibrator 3C84 (left) and galactic maser W3(OH) (right).


Figure 3: Amplitude and phase as function of channels at the baseline Irbene - Torun. Date: 26 Apr 2018, frequency: 6662.49 $\mathrm{MHz}$, calibrator 3C84 (left) and galactic maser $\mathrm{W} 3(\mathrm{OH})$ (right).

\section{Conclusions}

After modernization carried out during the years 2014 and 2015 and the development of the appropriate data reduction program, both of the VIRAC radio telescopes are suitable for VLBI and spectral line observations in the frequency range 4.5-8.8 GHz (starting with 2019 also in 1.6 $\mathrm{GHz}$ ). Furthermore, excluding other possible fields of research for these telescopes to be used in, both radio telescopes may be utilized as a small baseline interferometer for exact measurements of coordinates and sizes of maser sources. However, it is necessary to add one or two additional radio telescopes (e.g. Torun or Onsala) in order to monitor motion dynamics of maser sources with characteristic distances tens of mas between places of origin of different spectral features.

The successful VLBI experiment conducted in the baseline Irbene-Torun, which was scheduled and processed by VIRAC team, shows the ability of VIRAC correlation centre to conduct and correlate small-scale interferometric tasks.

\section{Acknowledgements}

Work is partially financed by Ventspils University of Applied Sciences project "Research of the possibility of creating a small VLBI array and radio astronomical data processing centre in VIRAC (TorIn); June - December 2018.

\section{References}

[1] V. Bezrukovs. Time and frequency synchronization on the VIRAC Radio telescope RT-32. Latvian J. Phys. Techn. Sci., (2016) vol. 53. 
[2] K. Skirmante, V. Bezrukovs, N. Jekabsons, I. Shmeld. Preparation of the VIRAC Radio Telescope RT32 for E-VLBI Observations. Baltic Astronomy, (2013) vol. 22.

[3] N.R. Vandenberg et al. Automation of the MARK III Field System, (1980).

[4] J. Jekabsons, D. Kotlere, M. Nechaeva. Mathematical and Algorithmic Description of Software Correlator for Space Debris Data Processing in VIRAC. European Space Surveillance conf, (2011).

[5] A. Keimpem, et al. The SFXC software correlator for Very Long Baseline Interferometry: Algorithms and Implementation. Experimental Astronomy, (2015).

[6] J. Steinbergs, F. Kamisevs, K. Skirmante, K. Automated Correlation System for radio astronomical data processing in VIRAC. Space Review (2018) vol.5. 